\title{
A Dense Companion to the Short-period Millisecond Pulsar Binary PSR J0636+5128
}

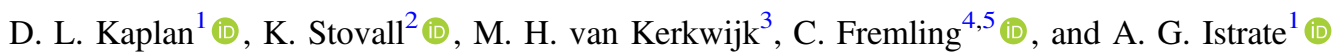 \\ ${ }^{1}$ Center for Gravitation, Cosmology and Astrophysics, Department of Physics, University of Wisconsin-Milwaukee, P.O. Box 413, \\ Milwaukee, WI 53201, USA; kaplan@uwm.edu \\ ${ }^{2}$ National Radio Astronomy Observatory, 1003 Lopezville Road, Socorro, NM 87801, USA \\ ${ }^{3}$ Department of Astronomy \& Astrophysics, University of Toronto, 50 Saint George Street, Toronto, ON M5S 3H4, Canada \\ ${ }^{4}$ The Oskar Klein Centre, Department of Astronomy, Stockholm University, AlbaNova, SE-10691 Stockholm, Sweden \\ ${ }^{5}$ Cahill Center for Astrophysics, California Institute of Technology, Pasadena, CA 91125, USA \\ Received 2018 June 28; revised 2018 July 19; accepted 2018 July 20; published 2018 August 27
}

\begin{abstract}
PSR J0636 +5128 is a millisecond pulsar in one of the most compact pulsar binaries known, with a 96 minute orbital period. The pulsar mass function suggests a very low mass companion, similar to that seen in so-called "black widow" binaries. Unlike in most of those, however, no radio eclipses by material driven off from the companion were seen leading to the possibility that the companion was a degenerate remnant of a carbon-oxygen white dwarf. We report the discovery of the optical counterpart of its companion in images taken with the Gemini North and Keck I telescopes. The companion varies between $r^{\prime}=25$ and $r^{\prime}=23$ on the 96 minute orbital period of the binary, caused by irradiation from the pulsar's energetic wind. We modeled the multicolor light curve using parallax constraints from pulsar timing and determine a companion mass of $(1.71 \pm 0.23) \times 10^{-2} M_{\odot}$, a radius of $(7.6 \pm 1.4) \times 10^{-2} R_{\odot}$, and a mean density of $54 \pm 26 \mathrm{~g} \mathrm{~cm}^{-3}$, all for an assumed neutron star mass of $1.4 M_{\odot}$. This makes the companion to PSR J0636+5128 one of the densest of the "black widow" systems. Modeling suggests that the composition is not predominantly hydrogen, perhaps due to an origin in an ultracompact X-ray binary.
\end{abstract}

Key words: binaries: general - pulsars: individual (PSR J0636+5128)

\section{Introduction}

PSR J0636+5128 (also called PSR J0636+5129) was discovered as part of the Green Bank North Celestial Cap pulsar survey (Stovall et al. 2014). It was particularly notable for its short orbital period of only $P_{B}=96$ minutes: only PSR J1311-3430 (Pletsch et al. 2012) and PSR J0024-7203R (Freire et al. 2017) have shorter orbits, and only by 2.2 minutes and $36 \mathrm{~s}$, respectively. It also has a rather low mass companion, with a minimum mass of about $7.4 M_{J}\left(7 \times 10^{-3} M_{\odot}\right.$, assuming a pulsar mass of $\left.M_{p}=1.4 M_{\odot}\right)$. This puts it into the range of "black widow" systems (Fruchter et al. 1988a, 1988b; Kulkarni et al. 1988; Roberts 2013), where a very low mass $\left(\lesssim 10^{-2} M_{\odot}\right)$ companion is in a tight orbit with an energetic pulsar. Typically eclipses are seen where ionized material driven off the companion delays and blocks the radio pulses, although this can be dependent on inclination. Systems also often have variations in their timing parameters suggestive of orbital interactions (e.g., Applegate \& Shaham 1994; Arzoumanian et al. 1994; Stappers et al. 1998; Pletsch \& Clark 2015; Shaifullah et al. 2016). Initially, no eclipses or timing variations were seen from PSR J0636+5128 (Stovall et al. 2014), leading to the suggestion that it was instead an inert, degenerate companion similar to that inferred in the PSR J1719-1438 system (Bailes et al. 2011), which has a mass of $\sim 1 M_{J}$ and a minimum mean density of $23 \mathrm{~g} \mathrm{~cm}^{-3}$. However, some black widows show no eclipses or other timing variations (e.g., Hessels et al. 2011; Bochenek et al. 2015; Arzoumanian et al. 2018), so further investigation of the nature of the companion to PSR J0636+5128 was necessary.

Such distinctions matter because the question of density is used as a proxy for composition, which is itself used to understand the formation mechanism for black widow and similar systems. The canonical model is that they evolve from low-mass X-ray binary
(LMXB) systems that move to tight orbits and lose considerable mass through accretion, ejection, and ablation (e.g., Ergma \& Fedorova 1992; Podsiadlowski et al. 2002; Benvenuto et al. 2012; Chen et al. 2013). In contrast, some systems may evolve from ultracompact X-ray binaries (UCXBs) consisting of a neutron star accreting from a degenerate white dwarf donor in a compact (orbital period of order an hour) binary (Deloye \& Bildsten 2003; van Haaften et al. 2012a, 2012b; Sengar et al. 2017). The binary companion would then have primarily a carbon/oxygen (if originally more massive) or helium composition, compared with hydrogen in the LMXB scenario, and these can be distinguished at some level through estimates of density (e.g., Tang et al. 2014; Spiewak et al. 2018). A lower limit for density is possible for these systems by the orbital period-mean density relation (e.g., Frank et al. 2002) constraining the density of the Roche lobe. If a companion can be identified, then further constraints are possible through estimates of the Roche-lobe filling fraction or companion radius (e.g., Tang et al. 2014; Spiewak et al. 2018). In the case of PSR J1719-1438, such an identification was hampered by its distance (dispersion measure distance of $1.2 \mathrm{kpc}$ ) and the crowded field at low Galactic latitude. However, for PSR J0636+5128 the higher Galactic latitude makes the search more promising.

Since its discovery, PSR J0636+5128 has been timed regularly (on a roughly monthly basis) as part of the North American Nanohertz Observatory for Gravitational Waves (NANOGrav; Arzoumanian et al. 2018). This data set includes a detection of a marginal timing parallax $\varpi=0.88 \pm 0.30$ mas (note that this implies a considerably larger distance than that determined in Stovall et al. 2014 and is not consistent with the previous value ${ }^{6}$ ) and a statistically significant orbital period

\footnotetext{
6 The explanation for the difference is likely the longer, higher-quality data span as well as better separation of secular trends in the dispersion measure from periodic (parallax) trends.
} 
derivative $\dot{P}_{B}=(2.5 \pm 0.3) \times 10^{-12}\left(P_{B} / \dot{P}_{B} \approx 77 \mathrm{Myr}\right)$. As in other black widow systems this orbital period derivative is unlikely to come from gravitational radiation, as it is of the wrong sign and two orders of magnitude too large: general relativity predicts $\dot{P}_{B}^{\mathrm{GR}}=-4.3 \times 10^{-14}$ assuming the nominal masses found below (e.g., Lorimer \& Kramer 2012). Instead, it likely reflects some mass loss or other orbital interaction in the system. In this paper, we report on the identification of the optical counterpart from observations with Gemini North and Keck I. Moreover, we measure significant orbital modulation coming from irradiation by the pulsar and use this to estimate the inclination and radius of the companion. Note that after submission of this manuscript, we became aware of Draghis \& Romani (2018), who combine our archival Gemini data with their own near-infrared imaging to study the companion of PSR J0636 +5128 . Our analysis of the light curve is broadly consistent with that of Draghis \& Romani (2018).

\section{Observations and Reduction}

We observed PSR J0636+5128 with the Gemini MultiObject Spectrograph (GMOS; Hook et al. 2004) on the $8.1 \mathrm{~m}$ Frederick C. Gillett Gemini North telescope on Mauna Kea in Hawaii. Observations consisted of $10 \times 420 \mathrm{~s}$ with the $g^{\prime}$ filter and $9 \times 420 \mathrm{~s}$ with the $r^{\prime}$ filter on the night of 2014 December 21 , spanning 69 minutes ( 0.7 orbits) in $g^{\prime}$ and 61 minutes ( 0.6 orbits) in $r^{\prime}$. The detector was binned $2 \times 2$ for a plate scale of $0.15 \mathrm{pixel}^{-1}$. Data were reduced using the GMOS pipeline (Shaw 2016). The airmass ranged from 1.2 to $1.3\left(g^{\prime}\right)$ and 1.3 to $1.5\left(r^{\prime}\right)$, while the seeing was about $1 . \prime 0\left(g^{\prime}\right)$ and 0 ." $9\left(r^{\prime}\right)$.

We also observed PSR J0636+5128 with the Low-Resolution Imaging Spectrometer (LRIS; Oke et al. 1995; Rockosi et al. 2010) on the $10 \mathrm{~m}$ Keck I telescope on Mauna Kea in Hawaii. Observations consisted of $13 \times 300 \mathrm{~s}$ using the red side $^{7}$ of the instrument and the $i$ filter on the night of 2018 January 18, spanning 87 minutes ( 0.9 orbits). The airmass ranged from 1.2 to 1.4 , and the seeing was about $1 . ! 9$. Data were reduced using the LPIPE package.

Once the raw images were reduced, we made sure that they were registered astrometrically by comparing with stars from the Pan STARRS $3 \pi$ survey (PS1; Chambers et al. 2016). We easily identified a variable optical source at the radio timing position of the pulsar (Figure 1). We measured fluxes (rejecting cosmic rays with a simple threshold) in a single aperture with a constant radius for each instrument/filter combination that was close to the seeing FWHM on 11 selected stars from PS1 and also the timing position of the pulsar. These stars were selected to be bright, well isolated, and not saturated. All of the reference stars were used to determine relative zero points within each filter, and absolute photometry was referenced to the star PSO J063600.942 +512838.878 , chosen because it was bright, nearby, and not in a crowded region; attempts to use an ensemble for more accurate absolute photometry had difficulties regardless of whether the photometric standard was PS1, the Sloan Digital Sky Survey (Abolfathi et al. 2018), or others. Based on observations of the ensemble of reference stars we believe the relative photometry to be accurate to about 0.02 mag

\footnotetext{
7 Observations using the blue side with the $g^{\prime}$ filter were processed, but the signal to noise was very low (best detections had a signal to noise of 3.5 , compared to 17 for GMOS) and the results were consistent with the GMOS data. Therefore, we use the GMOS $g^{\prime}$-band data exclusively.
}

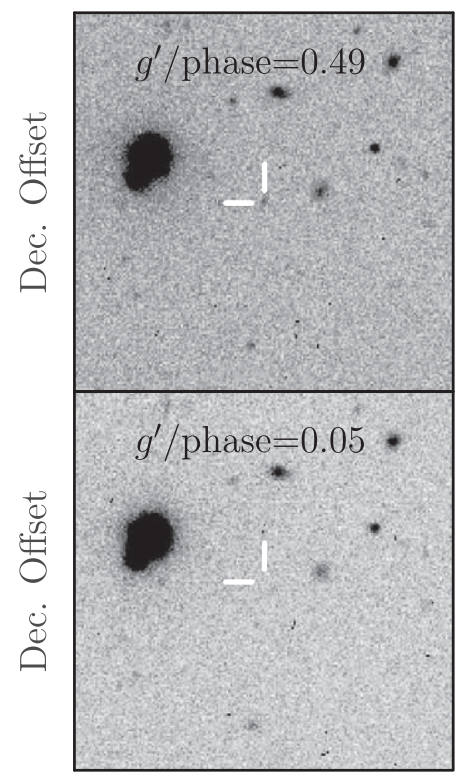

RA Offset

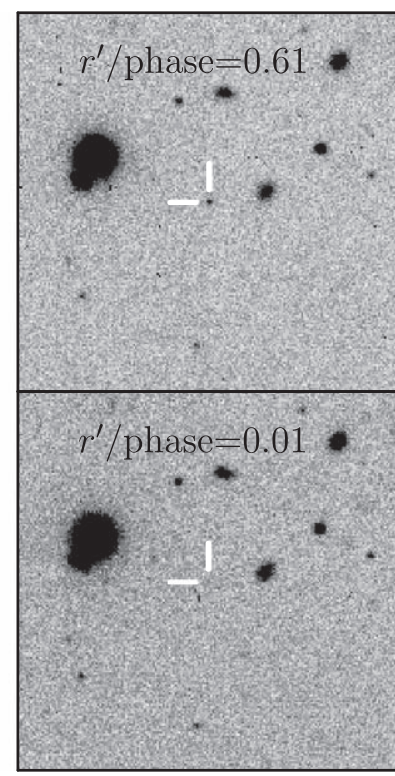

RA Offset
Figure 1. Gemini North GMOS images of PSR J0636+5128. We show the $g^{\prime}$ band (left) and $r^{\prime}$ band (right) for two different orbital phases, close to photometric maximum/phase of 0.5 (top) and close to photometric minimum/ phase of 0 (bottom). The images are $60^{\prime \prime}$ on a side, with north up and east to the left. The tick marks indicate the position of PSR J0636+5128.

$\left(g^{\prime}\right.$ and $\left.r^{\prime}\right)$ or $0.01 \mathrm{mag}(i)$. The absolute photometry is likely to only be accurate to about $0.1 \mathrm{mag}$, since we referenced it to a single star and do not include color terms relating to different photometric systems.

Finally, we corrected the image times to the solar system barycenter using routines in astropy and computed the orbital phase of the midpoint of each image.

\section{Light Curve Fitting}

We modeled the $g^{\prime}-, r^{\prime}$, , and $i$-band light curves of PSR J0636+5128 using Icarus (Breton et al. 2012). The model consisted of a binary with possible irradiation, ellipsoidal modulation, and Doppler boosting. We assumed corotation of the companion and took the gravity darkening coefficient to be 0.08 (appropriate for convective envelopes, which is likely the case in the effective temperature range that we found). The free parameters are the inclination $i$, Rochelobe filling factor $f$, backside temperature $T_{\text {night }}$, irradiated temperature excess $T_{\text {irr }}$, extinction $A_{V}$, and parallax $\varpi$; we held the neutron star mass to be fixed during the fitting. The irradiated temperature $T_{\text {irr }}$ is related to the frontside temperature $T_{\text {day }}$ (facing the pulsar) and the backside temperature $T_{\text {night }}$ by $T_{\text {day }}^{4}=T_{\text {night }}^{4}+T_{\text {irr }}^{4}$. For the inclination we used a prior distribution that was flat in $\cos i$; the priors for $f, T_{\text {night }}$, and $T_{\text {irr }}$ were uniform over $[0,1],[0,20,000] \mathrm{K}$, and $[0,20,000] \mathrm{K}$, respectively. We used a prior distribution for $A_{V}$ informed by the three-dimensional Galactic extinction model of Green et al. (2018), which gives $A_{V}=0.25 \pm 0.06 \mathrm{mag}$ at the nominal distance of $1.2 \mathrm{kpc}$. For the parallax we used a normal prior determined by the radio timing observations, $\mathcal{N}(0.88$ mas, 0.30 mas). Finally, we added an additional prior with $p(\varpi) \propto \varpi^{-4}$ to account for Lutz-Kelker bias (Lutz \& Kelker 1973; Verbiest et al. 2010) in this low-significance measurement. We note that underlying this prior is an 
Table 1

MCMC Light Curve Fit Results

\begin{tabular}{lccc}
\hline \hline Parameter & $M_{p}=1.4 M_{\odot}$ & $M_{p}=1.8 M_{\odot}$ & $M_{p}=2.0 M_{\odot}$ \\
\hline Fitted Parameters & & & \\
\hline$i{\text { degree })^{\mathrm{a}}}^{\mathrm{a}}$ & $24.3 \pm 3.5$ & $24.5 \pm 3.8$ & $24.6 \pm 4.2$ \\
$f^{\mathrm{b}}$ & $0.75 \pm 0.20$ & $0.75 \pm 0.19$ & $0.74 \pm 0.19$ \\
$T_{\text {night }}(\mathrm{K})^{\mathrm{c}}$ & $1643 \pm 1561$ & $1709 \pm 1638$ & $1706 \pm 1758$ \\
$T_{\text {irr }}(\mathrm{K})^{\mathrm{c}}$ & $4671 \pm 324$ & $4668 \pm 330$ & $4641 \pm 346$ \\
$\varpi(\mathrm{mas})^{\mathrm{d}}$ & $0.90 \pm 0.21$ & $0.86 \pm 0.19$ & $0.84 \pm 0.18$ \\
$A_{V}(\mathrm{mag})^{\mathrm{e}}$ & $0.25 \pm 0.09$ & $0.25 \pm 0.09$ & $0.24 \pm 0.09$ \\
\hline Derived Parameters & & & \\
\hline$\left.\rho_{c}(\mathrm{~g} \mathrm{~cm})^{-3}\right)^{\mathrm{f}}$ & $54 \pm 26$ & $53 \pm 26$ & $54 \pm 27$ \\
$M_{c}\left(10^{-2} M_{\odot}\right)^{\mathrm{f}}$ & $1.71 \pm 0.23$ & $1.94 \pm 0.28$ & $2.15 \pm 0.34$ \\
$R_{c}\left(10^{-2} R_{\odot}\right)^{\mathrm{f}}$ & $7.6 \pm 1.4$ & $7.9 \pm 1.4$ & $8.1 \pm 1.8$ \\
$\eta^{\mathrm{c}}$ & $0.18 \pm 0.05$ & $0.20 \pm 0.06$ & $0.22 \pm 0.07$ \\
$T_{\text {day }}(\mathrm{K})^{\mathrm{c}}$ & $4726 \pm 293$ & $4730 \pm 287$ & $4708 \pm 283$ \\
$d{(\mathrm{kpc})^{\mathrm{d}}}$ & $1.11 \pm 0.25$ & $1.17 \pm 0.26$ & $1.19 \pm 0.26$ \\
\hline
\end{tabular}

Notes. The values quoted here are the medians of the posterior probability distributions plus $1 \sigma$ confidence limits determined from the inner-quartile range scaled up by the square root of the reduced $\chi^{2}$.

${ }^{\text {a }}$ System inclination.

${ }^{b}$ Roche-lobe filling factor, defined as the radius of the companion facing the pulsar (the "nose") divided by the distance to the L1 Lagrange point.

${ }^{c}$ Backside temperature $T_{\text {night }}$ and irradiated temperature $T_{\text {irr }}$. We also give frontside temperature $T_{\text {day }}$, where $T_{\text {day }}^{4}=T_{\text {night }}^{4}+T_{\text {irr }}^{4}$ and irradiation efficiency $\eta$ where $\sigma T_{\text {irr }}^{4}=\eta \dot{E} / 4 \pi a^{4}$, with $\dot{E}=5.5 \times 10^{33} \mathrm{erg} \mathrm{s}^{-1}$ as the spin-down luminosity and $a$ the orbital separation. $\dot{E}$ is corrected for the proper motion (Shklovskii 1970) and assumes a moment of inertia of $10^{45} \mathrm{~g} \mathrm{~cm}^{2}$.

${ }^{\mathrm{d}}$ The parallax $\varpi$ and distance $d$.

e The $V$-band extinction.

f The companion mass $M_{c}$, companion radius $R_{c}$, and mean density $\rho_{c}$ determined from the assumed neutron star mass and inclination.

assumption of a constant space density for binaries like PSR J0636+5128, which is unlikely to hold. As for other types of MSP binaries, more realistic spatial distributions would likely lead one to infer somewhat smaller distances (e.g., Verbiest et al. 2012; Igoshev et al. 2016; Jennings et al. 2018). We allowed for an additional systematic offset that was free for each photometric band with an uncertainty of $0.1 \mathrm{mag}$, as discussed above. The $\chi^{2}$ from this band offset was added to the $\chi^{2}$ for the individual photometric points.

With this model we performed a Markov Chain Monte Carlo (MCMC) fit using the affine invariant MCMC ensemble sampler emcee (Foreman-Mackey et al. 2013). We started 400 "walkers" in the six-dimensional parameter space and allowed them to evolve for 100 steps to achieve "burn-in." We then reset the sampler and evolved for a further 1000 steps, saving all of the samples for a total of 40,000 MCMC samples.

In Table 1, we give the best-fit values of the parameters for three different assumed neutron star masses, taken to be the medians of the resulting posterior probability distribution functions. We give both the actual fitted parameters and derived parameters: the companion mass $M_{c}$ and radius $R_{c}$, the companion mean density $\rho_{c}$, and the irradiation efficiency $\eta$ defined by

$$
\sigma T_{\text {irr }}^{4}=\eta \frac{\dot{E}}{4 \pi a^{2}}
$$

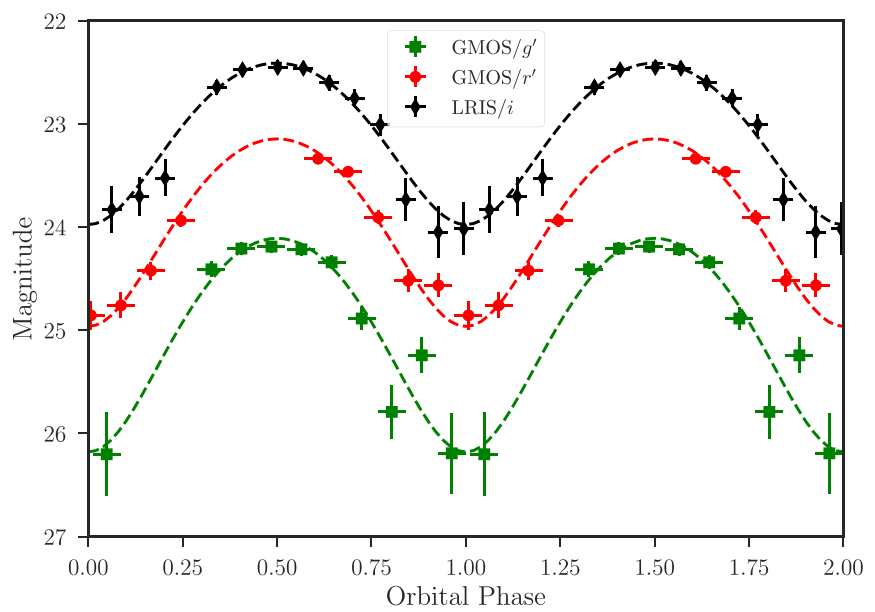

Figure 2. Best-fit Icarus light curve model for PSR J0636+5128 repeated twice for clarity, assuming a pulsar mass of $1.4 M_{\odot}$. Note that orbital phases of 0 and 0.5 correspond to conjunction. We show the GMOS $g^{\prime}$ and $r^{\prime}$ bands (green squares and red circles, respectively) and the LRIS $i$ band (black diamonds) together with the best-fit model light curves. The best-fit parameters are in Table 1.

where $\dot{E}$ is the spin-down luminosity of the pulsar, $a$ is the inferred orbital separation, and $\sigma$ is the Stefan-Boltzmann constant.

Overall, we were able to achieve a reasonable fit, and we show the best-fit light curve for $1.4 M_{\odot}$ in Figure 2 . The fit yields $\chi^{2}=48.4$ for 23 degrees of freedom, including systematic offset terms for each photometric band of $0.1 \mathrm{mag}$ each. We give the best-fit values and uncertainties (determined from the inner-quartile range, which is more robust to outliers than other methods) in Table 1. We have increased the uncertainties in the fitted parameters from Table 1 by the square root of the reduced $\chi^{2}$ to account for any underestimated uncertainties or modeling errors. The band offsets were small, consistent with our estimates of the systematic uncertainties. The dereddened color varies from $g^{\prime}-i=1.45$ at photometric maximum to $g^{\prime}-i=1.95$ at photometric minimum (assuming $A_{V}=0.25 \mathrm{mag}$ ), which implies changing from spectral type K4 to M0 (Covey et al. 2007) or effective temperatures ranging from 4600 to $3800 \mathrm{~K}$. We find no evidence for dramatic flares or other stochastic variations such as those seen in PSR J1311 -3430 (Romani et al. 2012). The photometric variability is dominated by irradiation of the companion by the pulsar (fractional amplitude of about $70 \%$ ), which has a period equal to the binary period. We see no evidence of ellipsoidal modulation (at twice the orbital period), which is consistent with the modest Roche-lobe filling of the companion. The ellipsoidal modulation should be at most about $2 \%$ (based on Breton et al. 2012), consistent with the amplitudes of fitted sinusoids. A final potential cause of periodic modulation is Doppler boosting (Maxted et al. 2000; Loeb \& Gaudi 2003; Zucker et al. 2007), which is at the orbital period, although at a different phase compared to irradiation, but despite the high inferred velocity for the companion (inferred radial velocity amplitude of $589 \mathrm{~km} \mathrm{~s}^{-1}$ ), this is only expected to be $1.6 \%$ even in the $g^{\prime}$ band, below our detection limits of $18 \%(2 \sigma)$.

The full fit results are shown in Figure 3. The best-fit values of $A_{V}$ and $\varpi$ agreed with the prior distributions. There is a small tail of inclinations that extends to high values, leading to a tail in the distributions of companion mass and density, but only about $10 \%$ of the probability has $i>40^{\circ}$. None of the other 


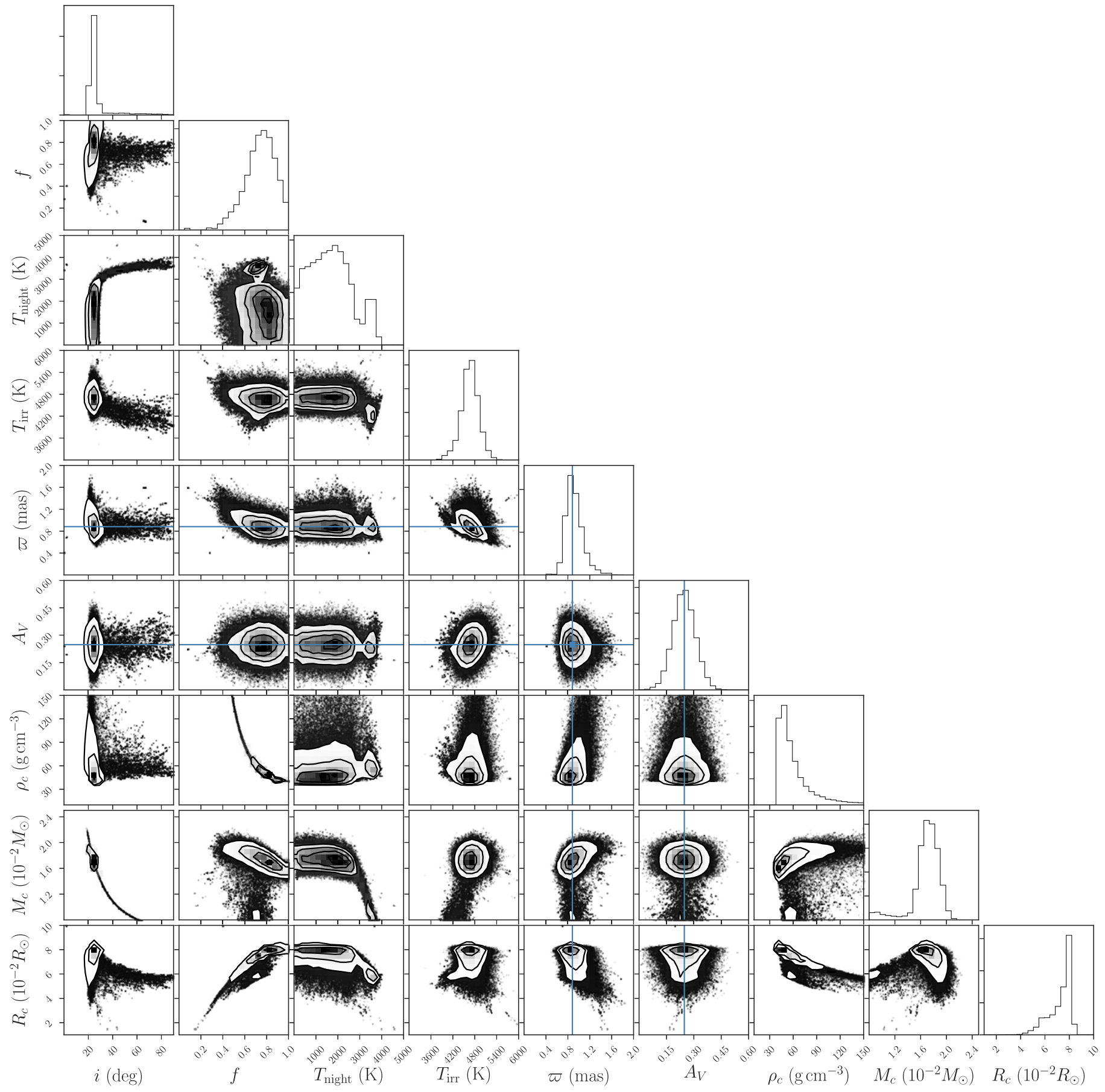

Figure 3. Corner plot showing the MCMC results for fitting a light curve to the data for PSR J0636+5128, assuming a pulsar mass of $1.4 M_{\odot}$. We show the distributions for the fitted parameters: inclination $i$, Roche-lobe filling fraction $f$, backside temperature $T_{\text {night }}$, irradiated temperature $T_{\text {irr }}$, parallax $\varpi$, extinction $A_{V}$. We also show distributions for three derived parameters: the mean density of the companion $\rho_{c}$, the mass of the companion $M_{c}$, and the radius of the companion $R_{c}$. For the parallax and extinction the vertical/horizontal lines show the means of the prior distributions determined from other sources.

fitted parameters had significant bimodalities or other issues. The backside temperature formally extends to low values, even as low as $0 \mathrm{~K}$. However, since the inclination is largely face-on, we never see just the backside of the companion, so the lowest area-averaged temperature is considerably higher, consistent with the colors above. Since a number of black widow systems have been observed to have high neutron star masses (e.g., van Kerkwijk et al. 2011; Romani et al. 2012; Schroeder \& Halpern 2014), our results are given both for a canonical neutron star mass of $1.4 M_{\odot}$ as well as higher values of $1.8 M_{\odot}$ and $2.0 M_{\odot}$. However, we do not see a significant shift in our fit results for those other values, as $M_{c}$ can just scale up along with a small increase in distance to compensate. In what follows, we will primarily discuss the results for $1.4 M_{\odot}$.

\section{Discussion and Conclusions}

The observed orbital period derivative $\dot{P}_{B}$ is of the wrong sign and two orders of magnitude too large to be explained by emission of gravitational radiation. Instead, we examine 


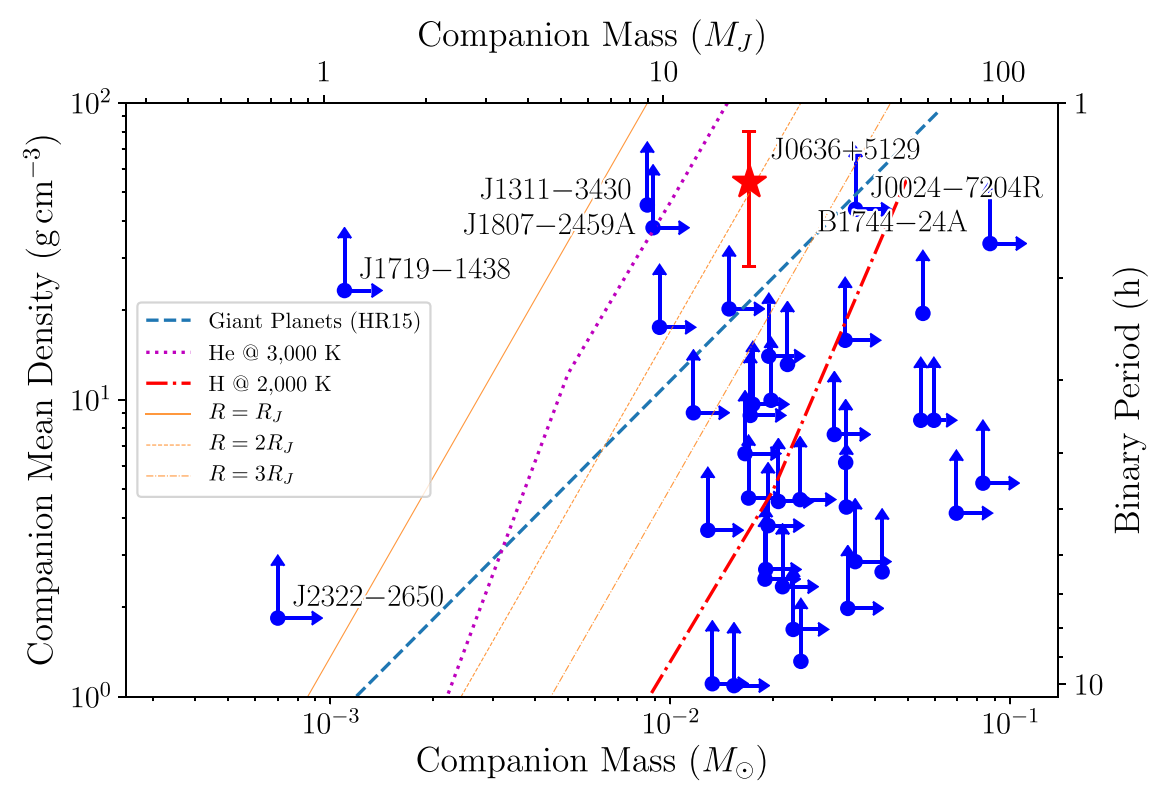

Figure 4. Companion mass vs. companion mean density for all "ultra-light" systems in the ATNF pulsar catalog (Manchester et al. 2005, 2016). For the majority of systems, densities are from the orbital period-mean density relation only (Frank et al. 2002), and the companion masses are the minimum companion masses assuming a $1.4 M_{\odot}$ neutron star. For a limited number of systems with published inclination constraints we have updated the companion masses: PSRs J0023+0923, J2256 -1024, J1810+1744, and J2215+5135 from Breton et al. (2013); PSR J2129-0429 from Bellm et al. (2016); PSR J1301+0833 from Romani et al. (2016); PSR B1957+20 from Reynolds et al. (2007); PSR J2051-0827 from Stappers et al. (1999); PSR J1953+1846A from Cadelano et al. (2015); PSR J1544+4937 from Tang et al. (2014); and PSR J1311-3430 from Romani et al. (2015). For PSR J0636+5128 (star) the numbers are from this paper. Select systems are labeled. The mass uncertainties correspond to the range of inclinations from $60^{\circ}$ to $90^{\circ}$, while the density uncertainties are a factor of 1.5 . We also plot the empirical fit to giant planets and brown dwarfs from Hatzes \& Rauer (2015; dashed line), models of a helium white dwarf remnant at an effective temperature of $3000 \mathrm{~K}$ (dotted line), and a hydrogen brown dwarf at $2000 \mathrm{~K}$ (dotted-dashed line). For reference, we also show sources with radii of 1, 2, and 3 times that of Jupiter (thin solid, dashed, and dotted-dashed lines).

whether or not it could be caused by mass loss from the system. First, we correct $\dot{P}_{B}$ (and $\dot{P}$ ) for the Shklovskii (1970) effect using our distance estimate and find that only a $3 \%$ correction is needed. Scaling the mass loss with the orbital period change,

$$
\dot{M}_{c} \sim\left(M_{c}+M_{p}\right) \frac{\dot{P}_{B}}{P_{B}},
$$

we find $\dot{M}_{c} \sim 10^{-8} M_{\odot} \mathrm{yr}^{-1}$. This is a plausible amount of mass loss for removing the majority of the mass of the companion in considerably less than a Hubble time after the end of mass transfer. However, it is four orders of magnitude larger than the mass-loss rate expected for pulsar irradiation (Stevens et al. 1992), although the irradiation efficiency is very similar to those found by Breton et al. (2013) for a number of other systems. Instead, $\dot{P}_{B}$ could originate in secular orbit interactions such as those seen in other black widow systems (e.g., Applegate \& Shaham 1994; Arzoumanian et al. 1994; Stappers et al. 1998). Further timing to search for higher-order derivatives would be conclusive. Unlike in other black widow systems, radio eclipses have not been detected from this system (Stovall et al. 2014), but can be understood by the relatively face-on inclination determined above (also see Draghis \& Romani 2018).

As discussed in Stovall et al. (2014), the minimum mean density inferred for the companion of PSR J0636+5128 is about $43 \mathrm{~g} \mathrm{~cm}^{-3}$, almost a factor of two larger than that inferred for the companion of PSR J1719-1438. This estimate assumes Roche-lobe filling: our smaller filling factor implies an even higher companion density of $\approx 54 \mathrm{~g} \mathrm{~cm}^{-3}$.

Our estimates for the companion's mass and radius place it right in the region predicted by Deloye \& Bildsten (2003) for systems with orbital periods of about 90 minutes. It is slightly smaller and denser than giant planets in this mass range (e.g., Hatzes \& Rauer 2015), which have densities $\approx 20 \mathrm{~g} \mathrm{~cm}^{-3}$, but this could be a combination of a different composition (more C/O-rich, as suggested by Bailes et al. 2011 for PSR J1719 -1438 ), other internal differences, or just measurement error. We compare with models generated using Modules for Experiments in Stellar Astrophysics (MESA; Paxton et al. 2011, version 10398). Hydrogen models are based on the brown_dwarf test_suite case. The models with helium composition were created starting from a white dwarf model of $0.35 M_{\odot}$ from the white_dwarf_models database and relaxing the mass until the desired mass is obtained. The helium model is shown at an effective temperature of $3000 \mathrm{~K}$ (similar to the upper limit on the backside temperature of PSR J0636+5128), while the hydrogen model is at $2000 \mathrm{~K}$ since they typically started at temperatures cooler than $3000 \mathrm{~K}$; a higher temperature would tend to decrease the density of the hydrogen models, leading to a worse match. Both of those model tracks are similar and largely parallel to a model at a constant radius, which is to be expected since sources have a constant radius for a wide span of mass in this range owing to the transition from degeneracy support to Coulomb support. At an effective temperature of $3000 \mathrm{~K}$, PSR J0636+5128 appears to have a composition with somewhat higher density than pure hydrogen (similar to helium), which suggests that it could be the remnant of a helium white dwarf, perhaps indicating a UCXB origin (Sengar et al. 2017).

Overall, as shown in Figure 4, PSR J0636+5128 appears to have one of the highest mean densities of any black widow system. However, the majority of densities are lower limits as they assume the system to be Roche-lobe filling, and a number 
of systems (especially those in globular clusters) do not have direct constraints. For instance, PSR J1544+4937 could have a density as high as $500 \mathrm{~g} \mathrm{~cm}^{-3}$ (suggesting an origin in a UCXB system), although the unconstrained distance means that it could also be a factor of 20 smaller (Tang et al. 2014).

In terms of previous evolution, our estimate of the mean density of $54 \mathrm{~g} \mathrm{~cm}^{-3}$ is consistent with the remnant of a helium-core white dwarf that has been ablated, evaporated, and/or accreted by the pulsar. It is possible the same holds for the companion to PSR J1719-1438, i.e., that it has a helium composition instead of the carbon-rich one favored by Bailes et al. (2011). If so, it would have a similar radius, since for these masses radius is predicted to be nearly independent of mass. If it also were equally hot, it would have been seen by Bailes et al. (2011), since the distances are similar as well and there is only $\sim 1 \mathrm{mag}$ of excess extinction. At first glance that might suggest the companion of PSR J1719-1438 is in fact smaller and thus made of denser material. However, the irradiation in this source is at least four times lower than for PSR J0636 +5128 , which would lead to a $\gtrsim 30 \%$ decrease in the irradiated temperature and therewith to a $\sim 1.5 \mathrm{mag}$ decrease in maximum companion optical brightness. This would be consistent with the non-detection of Bailes et al. (2011).

Unfortunately, PSR J0636+5128 is somewhat faint for optical spectroscopy, which could be used to determine the mass ratio and, in conjunction with modeling such as that presented here, determine the neutron star mass (van Kerkwijk et al. 2011; Romani et al. 2012). This is especially true since observations would need to cover only a small fraction of the orbital period in order to not suffer too much orbital smearing: alternate techniques such as "trailed" spectroscopy (e.g., Romani et al. 2015) are possible, but at two magnitudes fainter than PSR J1311-3430 it will still be difficult.

Partially based on observations obtained at the Gemini Observatory under program GN-2014B-Q-81 (PI: Stovall), which is operated by the Association of Universities for Research in Astronomy, Inc., under a cooperative agreement with the NSF on behalf of the Gemini partnership: the National Science Foundation (United States), the National Research Council (Canada), CONICYT (Chile), Ministerio de Ciencia, Tecnología e Innovación Productiva (Argentina), and Ministério da Ciência, Tecnologia e Inovação (Brazil). IRAF is distributed by the National Optical Astronomy Observatory, which is operated by the Association of Universities for Research in Astronomy (AURA) under a cooperative agreement with the National Science Foundation. Some data presented herein were obtained at the W. M. Keck Observatory, which is operated as a scientific partnership among the California Institute of Technology, the University of California and the National Aeronautics and Space Administration. The Observatory was made possible by the generous financial support of the W. M. Keck Foundation. The authors wish to recognize and acknowledge the very significant cultural role and reverence that the summit of Mauna Kea has always had within the indigenous Hawaiian community. We are most fortunate to have the opportunity to conduct observations from this mountain. Support for D.L.K. and K.S. was provided by the NANOGrav NSF Physics Frontiers Center award number 1430284. A.G.I. acknowledges support from the
NASA Astrophysics Theory Program through NASA grant NNX13AH43G.

Facilities: Gemini North (GMOS), Keck:I (LRIS).

Software: Astropy (Astropy Collaboration et al. 2013), corner (Foreman-Mackey 2016), emcee (Foreman-Mackey et al. 2013) Icarus (https://github.com/bretonr/Icarus), LPIPE (http://www.astro.caltech.edu/ dperley/programs/lris/manual. txt), MESA (Paxton et al. 2011), photutils (Bradley et al. 2017).

\section{ORCID iDs}

D. L. Kaplan (iD https://orcid.org/0000-0001-6295-2881

K. Stovall (i) https://orcid.org/0000-0002-7261-594X

C. Fremling (iD https://orcid.org/0000-0002-4223-103X

A. G. Istrate (i) https://orcid.org/0000-0002-8811-8171

\section{References}

Abolfathi, B., Aguado, D. S., Aguilar, G., et al. 2018, ApJS, 235, 42 Applegate, J. H., \& Shaham, J. 1994, ApJ, 436, 312

Arzoumanian, Z., Brazier, A., Burke-Spolaor, S., et al. 2018, ApJS, 235, 37 Arzoumanian, Z., Fruchter, A. S., \& Taylor, J. H. 1994, ApJL, 426, 85

Astropy Collaboration, Robitaille, T. P., Tollerud, E. J., et al. 2013, A\&A, 558, A33

Bailes, M., Bates, S. D., Bhalerao, V., et al. 2011, Sci, 333, 1717

Bellm, E. C., Kaplan, D. L., Breton, R. P., et al. 2016, ApJ, 816, 74 Benvenuto, O. G., De Vito, M. A., \& Horvath, J. E. 2012, ApJL, 753, L33

Bochenek, C., Ransom, S., \& Demorest, P. 2015, ApJL, 813, L4

Bradley, L., Sipocz, B., Robitaille, T., et al. 2017, astropy/photutils, v0.4, Zenodo, doi:10.5281/zenodo.1039309

Breton, R. P., Rappaport, S. A., van Kerkwijk, M. H., \& Carter, J. A. 2012, ApJ, 748, 115

Breton, R. P., van Kerkwijk, M. H., Roberts, M. S. E., et al. 2013, ApJ, 769, 108

Cadelano, M., Pallanca, C., Ferraro, F. R., et al. 2015, ApJ, 807, 91

Chambers, K. C., Magnier, E. A., Metcalfe, N., et al. 2016, arXiv:1612.05560

Chen, H.-L., Chen, X., Tauris, T. M., \& Han, Z. 2013, ApJ, 775, 27

Covey, K. R., Ivezić, Ž, Schlegel, D., et al. 2007, AJ, 134, 2398

Deloye, C. J., \& Bildsten, L. 2003, ApJ, 598, 1217

Draghis, P., \& Romani, R. W. 2018, ApJL, 862, L6

Ergma, E., \& Fedorova, A. V. 1992, A\&A, 265, 65

Foreman-Mackey, D. 2016, JOSS, 1, 24

Foreman-Mackey, D., Hogg, D. W., Lang, D., \& Goodman, J. 2013, PASP, 125,306

Frank, J., King, A., \& Raine, D. J. 2002, Accretion Power in Astrophysics (3rd ed.; Cambridge: Cambridge Univ. Press)

Freire, P. C. C., Ridolfi, A., Kramer, M., et al. 2017, MNRAS, 471, 857 Fruchter, A. S., Gunn, J. E., Lauer, T. R., \& Dressler, A. 1988a, Natur, 334, 686 Fruchter, A. S., Stinebring, D. R., \& Taylor, J. H. 1988b, Natur, 333, 237

Green, G. M., Schlafly, E. F., Finkbeiner, D., et al. 2018, MNRAS, 478, 651 Hatzes, A. P., \& Rauer, H. 2015, ApJL, 810, L25

Hessels, J. W. T., Roberts, M. S. E., McLaughlin, M. A., et al. 2011, in AIP Conf. Ser. 1357, RADIO PULSARS: AN ASTROPHYSICAL KEY TO UNLOCK THE SECRETS OF THE UNIVERSE, ed. M. Burgay et al. (Melville, NY: AIP), 40

Hook, I. M., Jørgensen, I., Allington-Smith, J. R., et al. 2004, PASP, 116, 425 Igoshev, A., Verbunt, F., \& Cator, E. 2016, A\&A, 591, A123

Jennings, R. J., Kaplan, D. L., Chatterjee, S., Cordes, J. M., \& Deller, A. T. 2018, ApJ, in press (arXiv:1806.06076)

Kulkarni, S. R., Djorgovski, S., \& Fruchter, A. S. 1988, Natur, 334, 504

Loeb, A., \& Gaudi, B. S. 2003, ApJL, 588, L117

Lorimer, D. R., \& Kramer, M. 2012, Handbook of Pulsar Astronomy (Cambridge: Cambridge Univ. Press)

Lutz, T. E., \& Kelker, D. H. 1973, PASP, 85, 573

Manchester, R. N., Hobbs, G. B., Teoh, A., \& Hobbs, M. 2005, AJ, 129, 1993 Manchester, R. N., Hobbs, G. B., Teoh, A., \& Hobbs, M. 2016, yCat, 102043 Maxted, P. F. L., Marsh, T. R., \& North, R. C. 2000, MNRAS, 317, L41 Oke, J. B., Cohen, J. G., Carr, M., et al. 1995, PASP, 107, 375

Paxton, B., Bildsten, L., Dotter, A., et al. 2011, ApJS, 192, 3

Pletsch, H. J., \& Clark, C. J. 2015, ApJ, 807, 18

Pletsch, H. J., Guillemot, L., Fehrmann, H., et al. 2012, Sci, 338, 1314

Podsiadlowski, P., Rappaport, S., \& Pfahl, E. D. 2002, ApJ, 565, 1107 
Reynolds, M. T., Callanan, P. J., Fruchter, A. S., et al. 2007, MNRAS, 379,1117

Roberts, M. S. E. 2013, in IAU Symp. 291, Neutron Stars and Pulsars: Challenges and Opportunities after 80 years, ed. J. van Leeuwen (Cambridge: Cambridge Univ. Press), 127

Rockosi, C., Stover, R., Kibrick, R., et al. 2010, Proc. SPIE, 7735, 77350R

Romani, R. W., Filippenko, A. V., \& Cenko, S. B. 2015, ApJ, 804, 115

Romani, R. W., Filippenko, A. V., Silverman, J. M., et al. 2012, ApJL, 760, L36

Romani, R. W., Graham, M. L., Filippenko, A. V., \& Zheng, W. 2016, ApJ, 833,138

Schroeder, J., \& Halpern, J. 2014, ApJ, 793, 78

Sengar, R., Tauris, T. M., Langer, N., \& Istrate, A. G. 2017, MNRAS, 470, L6

Shaifullah, G., Verbiest, J. P. W., Freire, P. C. C., et al. 2016, MNRAS, 462, 1029

Shaw, R. A. 2016, GMOS Data Reduction Cookbook (Tucson, AZ: National Optical Astronomy Observatory)

Shklovskii, I. S. 1970, SvA, 13, 562
Spiewak, R., Bailes, M., Barr, E. D., et al. 2018, MNRAS, 475, 469

Stappers, B. W., Bailes, M., Manchester, R. N., Sandhu, J. S., \& Toscano, M. 1998, ApJL, 499, L183

Stappers, B. W., van Kerkwijk, M. H., Lane, B., \& Kulkarni, S. R. 1999, ApJL, 510, L45

Stevens, I. R., Rees, M. J., \& Podsiadlowski, P. 1992, MNRAS, 254, 19P

Stovall, K., Lynch, R. S., Ransom, S. M., et al. 2014, ApJ, 791, 67

Tang, S., Kaplan, D. L., Phinney, E. S., et al. 2014, ApJL, 791, L5

van Haaften, L. M., Nelemans, G., Voss, R., \& Jonker, P. G. 2012a, A\&A, 541, A22

van Haaften, L. M., Nelemans, G., Voss, R., Wood, M. A., \& Kuijpers, J. 2012b, A\&A, 537, A104

van Kerkwijk, M. H., Breton, R. P., \& Kulkarni, S. R. 2011, ApJ, 728, 95

Verbiest, J. P. W., Lorimer, D. R., \& McLaughlin, M. A. 2010, MNRAS, 405, 564

Verbiest, J. P. W., Weisberg, J. M., Chael, A. A., Lee, K. J., \& Lorimer, D. R. 2012, ApJ, 755, 39

Zucker, S., Mazeh, T., \& Alexander, T. 2007, ApJ, 670, 1326 